\title{
ROLE OF PRESENT AND FUTURE ATOMIC PARITY VIOLATION EXPERIMENTS IN PRECISION ELECTROWEAK TESTS 1
}

\author{
Jonathan L. Rosner 7 \\ Enrico Fermi Institute and Department of Physics \\ University of Chicago, 5640 S. Ellis Avenue, Chicago, IL 60637
}

(Received )

\begin{abstract}
Recent reanalyses of the atomic physics effects on the weak charge in cesium have led to a value in much closer agreement with predictions of the Standard Model. We review precision electroweak tests, their implications for upper bounds on the mass of the Higgs boson, possible ways in which these bounds may be circumvented, and the requirements placed upon accuracy of future atomic parity violation experiments by these considerations.
\end{abstract}

PACS Categories: 11.30.Er, 12.15.Ji, 12.15.Mm, 12.60.Cn

The successful unification of the weak and electromagnetic interactions [1] has been tested to the level of radiative corrections affected by the mass of the Higgs boson [2]. However, Peskin and Wells [3] have noted several contexts in which assumptions about electroweak symmetry breaking can be relaxed, leading to looser bounds on the Higgs boson mass. As one example, a small vacuum expectation value of a Higgs triplet [⿴囗 a can permit a Higgs boson mass in excess of $1 \mathrm{TeV}$. Specific models (e.g., [3, 5]) with this property have been constructed. Other related discussions may be found in [6, 7, 8].

Among the electroweak observables that play a role in precise tests of the radiative corrections in the theory, atomic parity violation plays a special role. Many types of new physics affect what are known as "oblique corrections," through vacuum polarization of the photon, $Z$, and $W$ bosons. These effects have been described by Peskin and Takeuchi [9] in terms of two parameters $S$ and $T$, upon which various observables depend linearly, with $S=T=0$ corresponding to "no new physics," given nominal values of the top quark and Higgs boson masses. The weak charge $Q_{W}$ measured in parity-violation experiments in such atoms as cesium [10, 11], bismuth [12], lead [13], and thallium [14, 15] is mainly sensitive to the variable $S$, with very small dependence on $T$ 116, 17, 18. Thus, atomic parity violation experiments can shed unique light on certain types of new physics which contribute to the parameter $S$ [19, 20, 21].

Atomic physics calculations have been carried out for such systems as cesium 22] and thallium [23. In 1999 the JILA-Boulder group reported measurements in cesium 111 that reduced uncertainties in previous calculations. This led to a resulting weak charge, $Q_{W}(\mathrm{Cs})=-72.06 \pm 0.28_{\text {expt }} \pm 0.34_{\text {theor }}=-72.06 \pm 0.46$ which represented a

\footnotetext{
${ }^{1}$ Enrico Fermi Institute preprint EFI 01-43, hep-ph/0109239. To be submitted to Physical Review D.

${ }^{2}$ rosner@hep.uchicago.edu
} 
considerable improvement with respect to previous values in this and other atoms. It was also more than two standard deviations away from the Standard Model prediction 16, 24] $Q_{W}(\mathrm{Cs})=-73.19 \pm 0.13$, leading to speculations [21, 25, 26] about possible sources of the discrepancy such as $Z^{\prime}$ bosons [27, 28] in extended gauge theories. No such bosons have been seen up to masses of about $600 \mathrm{GeV} / c^{2}$ [29].

Several recent contributions [30, 31, 32, 33] have re-evaluated atomic physics corrections in cesium, paying particular attention to the Breit interaction [34]. Our working average for these determinations will be $Q_{W}(\mathrm{Cs})=-72.2 \pm 0.8$. In the present paper we review the main electroweak observables affecting the mass of the Higgs boson, some possible ways in which upper bounds on this mass may be circumvented, and requirements placed upon accuracy of future atomic parity violation experiments by these considerations.

We begin with a brief review of the formalism of [9]. Electroweak radiative corrections may be divided into "oblique" and "direct" contributions. Oblique corrections (sensitive to all forms of new physics) enter through gauge boson vacuum polarization terms, and direct corrections include all other terms such as vertex and self-energy modifications. At lowest order, the $W$ mass $M_{W}$, the $Z$ mass $M_{Z}$, the electroweak couplings $g$ and $g^{\prime}$, the electric charge $e$, the weak mixing angle $\theta$, the Higgs doublet vacuum expectation value $v$, and the Fermi coupling constant $G_{F}=1.16637(1) \times 10^{-5}$ $\mathrm{GeV}^{-2}$ are related by

$$
e=g \sin \theta=g^{\prime} \cos \theta \quad, \quad \frac{G_{F}}{\sqrt{2}}=\frac{g^{2}}{8 M_{W}^{2}}=\frac{g^{2}+g^{\prime 2}}{8 M_{Z}^{2}}=\frac{1}{2 v^{2}}
$$

under the assumption that the only contribution to electroweak symmetry breaking comes from one or more Higgs doublets with vacuum expectation values $v_{i}$ satisfying $\sum_{i} v_{i}^{2}=v^{2}$. With $\alpha \equiv e^{2} / 4 \pi$ one then has

$$
M_{W}=\frac{\left(\pi \alpha / \sqrt{2} G_{F}\right)^{1 / 2}}{\sin \theta}, \quad M_{Z}=M_{W} / \cos \theta .
$$

Photon vacuum polarization effects change $\alpha^{-1}$ from its value of $\sim 137.036$ at $q^{2}=0$ to $128.933 \pm 0.021$ at $q^{2}=M_{Z}^{2}$ [35]. This important oblique correction is sensitive to all charged particles with masses less than $\mathcal{O}\left(M_{Z} / 2\right)$.

The next-most-important oblique correction arises from the large splitting between the top and bottom quark masses [36], violating a custodial $S U(2)$ symmetry [37] responsible for preserving the tree-level relation $M_{W}=M_{Z} \cos \theta$. As a result, an effect is generated equivalent to a Higgs triplet vacuum expectation value. The vacuum polarization diagrams with $W^{+} \rightarrow t \bar{b} \rightarrow W^{+}$and $Z \rightarrow(t \bar{t}, b \bar{b}) \rightarrow Z$ lead to a modification of the relation between $G_{F}$, coupling constants, and $M_{Z}$ for neutralcurrent exchanges:

$$
\frac{G_{F}}{\sqrt{2}}=\frac{g^{2}+g^{\prime 2}}{8 M_{Z}^{2}} \quad \rightarrow \quad \frac{G_{F}}{\sqrt{2}} \rho=\frac{g^{2}+g^{\prime 2}}{8 M_{Z}^{2}} \quad, \quad \rho \simeq 1+\frac{3 G_{F} m_{t}^{2}}{8 \pi^{2} \sqrt{2}} .
$$

The $Z$ mass is now related to the weak mixing angle by

$$
M_{Z}^{2}=\frac{\pi \alpha}{\sqrt{2} G_{F} \rho \sin ^{2} \theta \cos ^{2} \theta}
$$


where we have omitted some small terms logarithmic in $m_{t}$. A precise measurement of $M_{Z}$ now specifies $\theta$ only if $m_{t}$ is known, so $\theta=\theta\left(m_{t}\right)$ and hence $M_{W}^{2}=$ $\pi \alpha /\left(\sqrt{2} G_{F} \sin ^{2} \theta\right)$ is also a function of $m_{t}$.

To display dependence of electroweak observables on such quantities as the top quark and Higgs boson masses $m_{t}$ and $M_{H}$, we expand the observables about nominal values [2] calculated for specific $m_{t}$ and $M_{H}$. We thereby isolate the dependence on $m_{t}, M_{H}$, and new physics arising from oblique corrections associated with loops in the $W$ and $Z$ propagators. For $m_{t}=174.3 \mathrm{GeV}, M_{H}=100 \mathrm{GeV}$, the measured value of $M_{Z}$ leads to a nominal expected value of $\sin ^{2} \theta_{\text {eff }}=0.23140$. In what follows we shall interpret the effective value of $\sin ^{2} \theta$ as that measured via leptonic vector and axial-vector couplings: $\sin ^{2} \theta^{\mathrm{eff}} \equiv(1 / 4)\left(1-\left[g_{V}^{\ell} / g_{A}^{\ell}\right]\right)$.

Defining the parameter $T$ by $\Delta \rho \equiv \alpha T$, we find

$$
T \simeq \frac{3}{16 \pi \sin ^{2} \theta}\left[\frac{m_{t}^{2}-(174.3 \mathrm{GeV})^{2}}{M_{W}^{2}}\right]-\frac{3}{8 \pi \cos ^{2} \theta} \ln \frac{M_{H}}{100 \mathrm{GeV}} .
$$

The weak mixing angle $\theta$, the $W$ mass, and other electroweak observables now depend on $m_{t}$ and $M_{H}$.

The weak charge-changing and neutral-current interactions are probed under a number of different conditions, corresponding to different values of momentum transfer. In order to account for such effects we may replace the lowest-order relations between $G_{F}$, couplings, and masses by

$$
\frac{G_{F}}{\sqrt{2}}=\frac{g^{2}}{8 M_{W}^{2}}\left(1+\frac{\alpha S_{W}}{4 \sin ^{2} \theta}\right) \quad, \quad \frac{G_{F} \rho}{\sqrt{2}}=\frac{g^{2}+g^{\prime 2}}{8 M_{Z}^{2}}\left(1+\frac{\alpha S_{Z}}{4 \sin ^{2} \theta \cos ^{2} \theta}\right),
$$

where $S_{W}$ and $S_{Z}$ are coefficients representing variation with momentum transfer. Together with $T$, they express a wide variety of electroweak observables in terms of quantities sensitive to new physics. (The presence of such corrections was noted in [36].) The variable $U$ defined in [9] is equal to $S_{W}-S_{Z}$, while $S \equiv S_{Z}$.

Expressing the new physics effects in terms of deviations from nominal values of top quark and Higgs boson masses, we have the expression (5) for $T$, while contributions of Higgs bosons and of possible doublets of new degenerate fermions $U$ and $D$ to $S_{W}$ and $S_{Z}$, in a leading-logarithm approximation, are [38

$$
S_{W}=S_{Z}=\frac{1}{6 \pi}\left[\ln \frac{M_{H}}{100 \mathrm{GeV} / c^{2}}+\sum N_{c}\right]
$$

where $N_{c}$ is the number of colors of the new fermions, and the sum is taken over all such doublets. (See 38 for the case $m_{U} \neq m_{D}$.)

A degenerate heavy fermion doublet with $N_{c}$ colors thus contributes $\Delta S_{Z}=$ $\Delta S_{W}=N_{c} / 6 \pi$. For example, in a minimal dynamical symmetry-breaking ("technicolor") scheme, with a single doublet of $N_{c}=4$ fermions, one will have $\Delta S=$ $2 / 3 \pi \simeq 0.2$. This will turn out to be marginally acceptable under the condition that a small impurity of Higgs-triplet symmetry breaking is admitted, while many non-minimal schemes, with larger numbers of doublets, will be ruled out. 
The prediction $M_{Z}=M_{W} / \cos \theta$ is specific to the assumption that only Higgs doublets of $\mathrm{SU}(2)_{L}$ exist. $\left[\mathrm{SU}(2)_{L}\right.$ singlets which are neutral also have $Y=0$, and do not affect $W$ and $Z$ masses.] For a complex $Y=2$ triplet of the form

$$
\Phi \equiv\left[\begin{array}{c}
\Phi^{++} \\
\Phi^{+} \\
\Phi^{0}
\end{array}\right] \quad, \quad I_{3 L}=\left\{\begin{array}{c}
+1 \\
0 \\
-1
\end{array},\right.
$$

the contribution of $\left\langle\Phi^{0}\right\rangle=V_{1,-1} / \sqrt{2}$ to gauge boson masses (see, e.g., [39]) is

$$
M_{W}^{2}=\frac{g^{2}}{4}\left(v^{2}+2 V_{1,-1}^{2}\right), \quad M_{Z}^{2}=\left(\frac{g^{2}+g^{2}}{4}\right)\left(v^{2}+4 V_{1,-1}^{2}\right),
$$

so the ratio $\rho=\left(M_{W} / M_{Z} \cos \theta\right)^{2}$ is no longer 1 , but becomes

$$
\rho=\frac{v^{2}+2 V_{1,-1}^{2}}{v^{2}+4 V_{1,-1}^{2}} .
$$

This type of Higgs boson thus leads to $\rho<1$.

In the $Y=0$ triplet

$$
\Phi \equiv\left[\begin{array}{c}
\Phi^{+} \\
\Phi^{0} \\
\Phi^{-}
\end{array}\right] \quad, \quad I_{3 L}=\left\{\begin{array}{c}
+1 \\
0 \\
-1
\end{array},\right.
$$

if $\left\langle\Phi^{0}\right\rangle=V_{1,0} / \sqrt{2}$, we find by a similar calculation that

$$
M_{W}^{2}=\frac{g^{2}}{4}\left(v^{2}+4 V_{1,0}^{2}\right) \quad, \quad M_{Z}^{2}=\left(\frac{g^{2}+g^{\prime 2}}{4}\right) v^{2} .
$$

Here we predict

$$
\rho=1+\frac{4 V_{1,0}^{2}}{v^{2}},
$$

so this type of Higgs boson leads to $\rho>1$.

We now present a simplified analysis of present electroweak data in the $S, T$ framework which captures the essential elements. (See, e.g., [40] for a more complete version.) We shall assume $S_{W}=S_{Z}=S$. The present analysis is an update of [21], which may be consulted for further references. (See also [3].) We include atomic parity violation in cesium and thallium (as in [21]), the observed values of $M_{W}$ as measured at the Fermilab Tevatron and LEP-II, the leptonic width of the $Z$, the value of $\sin ^{2} \theta_{\text {eff }}$ as measured in various asymmetry experiments at the $Z$ pole in $e^{+} e^{-}$collisions, and the recent measurement by the $\mathrm{NuTeV}$ Collaboration [41] of a combination of neutrino and antineutrino neutral-current to charged-current cross section ratios $R_{\nu}$ and $R_{\bar{\nu}}$.

The inputs, their nominal values for $m_{t}=174.3 \mathrm{GeV}$ and $M_{H}=100 \mathrm{GeV}$, and their dependences on $S$ and $T$ are shown in Table ! . The value of $Q_{W}(\mathrm{Cs})$ in this table has been distilled from those in Table [1]. On the basis of the comment in Ref. 
Table I: Electroweak observables described in fit.

\begin{tabular}{ccc}
\hline Quantity & $\begin{array}{c}\text { Experimental } \\
\text { value }\end{array}$ & $\begin{array}{c}\text { Theoretical } \\
\text { value }\end{array}$ \\
\hline$Q_{W}(\mathrm{Cs})$ & $-72.2 \pm 0.8^{a)}$ & $-73.19^{b)}-0.800 S-0.007 T$ \\
$Q_{W}(\mathrm{Tl})$ & $-115.0 \pm 4.5^{c)}$ & $-116.8^{d)}-1.17 S-0.06 T$ \\
$M_{W}\left(\mathrm{GeV} / c^{2}\right)$ & $80.451 \pm 0.033^{e)}$ & $80.385^{f)}-0.29 S+0.45 T$ \\
$\Gamma_{\ell \ell}(Z)(\mathrm{MeV})$ & $83.991 \pm 0.087^{g)}$ & $84.011^{f)}-0.18 S+0.78 T$ \\
$\sin ^{2} \theta^{\mathrm{eff}}$ & $0.23152 \pm 0.00017^{g)}$ & $0.23140^{f)}+0.00362-0.00258 T$ \\
" $M_{W}$ " $\left(\mathrm{GeV} / c^{2}\right)$ & $80.136 \pm 0.084^{h)}$ & $80.385^{f)}-0.27 S+0.56 T$ \\
\hline
\end{tabular}

a) Weak charge in cesium [10, 11] incorporating recalculated atomic physics corrections [30, 31, 32, 33].

b) Calculation 16] incorporating electroweak corrections, updated in [24].

c) Weak charge in thallium [14, 15] incorporating atomic physics corrections [23].

d) Calculation incorporating electroweak corrections [43] .

e) Ref. 444] ${ }^{f)}$ Ref. [2]. ${ }^{g)}$ Ref. 455.

h) Based on NuTeV measurement of ratios $R_{\nu}$ and $R_{\bar{\nu}}$ (see text) 41].

[33] that other determinations have ignored a strong-field correction, we have taken as a central value that implied by Ref. [33]. The $\mathrm{NuTeV}$ data may be expressed as an effective measurement of the $W$ mass, with small corrections quoted in Ref. [41]. We use these corrections to arrive at the $S$ and $T$ dependences of " $M_{W}$ ". These supersede those quoted in Ref. [21], which were incorrectly inferred from an earlier $\mathrm{NuTeV}$ report 42.

We do not constrain the top quark mass; we shall display its effect on $S$ and $T$ explicitly. Each observable specifies a pair of parallel lines in the $S-T$ plane. The leptonic width mainly constrains $T ; \sin ^{2} \theta^{\text {eff }}$ provides a good constraint on $S$ with some $T$-dependence; and direct measurements of $M_{W}$ or values of " $M_{W}$ " implied by the $\mathrm{NuTeV}$ data lie in between. The atomic parity violation experiments constrain $S$ almost exclusively, but we shall see that they have little impact at their present level of sensitivity. Since the slopes are very different, the resulting allowed region is an ellipse, shown in Figure 1 (with the atomic parity violation data). The corresponding figure with those data omitted is almost identical, but shifted in central values by +0.01 unit in each of $S$ and $T$. The fits with and without the atomic parity violation data are compared in Table [II].

Figure 1 also shows predictions [3] of the standard electroweak theory. Nearly vertical lines correspond, from left to right, to Higgs boson masses $M_{H}=100,200$, $300,500,1000 \mathrm{GeV}$; drooping curves correspond, from top to bottom, to $+1 \sigma$, central, and $-1 \sigma$ values of $m_{t}=174 \pm 5.1 \mathrm{GeV}$.

In the standard model, the combined constraints of electroweak observables such as those in Table $\mathbb{1}$ and the top quark mass favor a very light Higgs boson, with most analyses favoring a value of $M_{H}$ so low that the Higgs boson should already 
Table II: Values of $Q_{W}(\mathrm{Cs})$ used to obtain the average in Table $\mathrm{\Psi}$.

\begin{tabular}{crc}
\hline Author(s) & Reference & $Q_{W}(\mathrm{Cs})$ \\
\hline Derevianko & 30 & $-72.61 \pm 0.28_{\text {expt }} \pm 0.73_{\text {theor }}$ \\
Kozlov et al. & 31 & $-72.5 \pm 0.7$ \\
Dzuba et al. & {$[32$} & $-72.42 \pm 0.28_{\text {expt }} \pm 0.74_{\text {theor }}$ \\
Milstein and Sushkov & {$[33$} & $\simeq-72.2$ \\
\hline
\end{tabular}

Table III: Comparison of fits with and without atomic parity violation data.

\begin{tabular}{ccc}
\hline & $S_{0}$ & $T_{0}$ \\
\hline APV data & $0.01 \pm 0.15$ & $0.00 \pm 0.15$ \\
No APV data & $0.02 \pm 0.15$ & $0.01 \pm 0.15$ \\
\hline
\end{tabular}

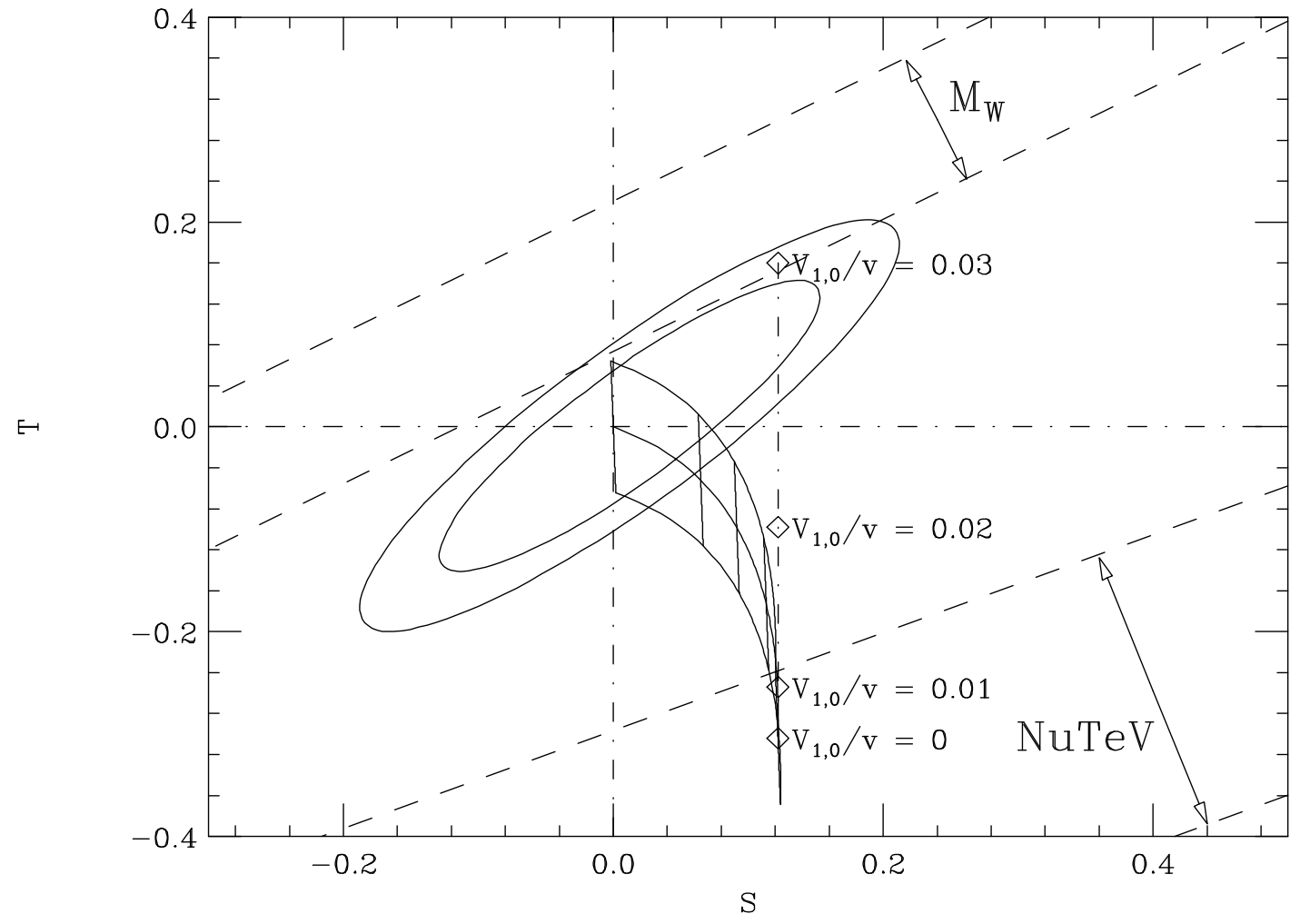

Figure 1: Regions of $68 \%$ (inner ellipse) and 90\% (outer ellipse) confidence level values of $S$ and $T$ based on the comparison of the theoretical and experimental electroweak observables shown in Table $\mathbb{\text { I, }}$, including atomic parity violation data (first two lines). Diagonal bands bounded by dashed lines correspond to $\pm 1 \sigma$ constraints associated with direct $M_{W}$ measurements (upper left) and with $\mathrm{NuTeV}$ measurements [41] of $R_{\nu}$ and $R_{\bar{\nu}}$ (lower right). Standard model predictions (solid nearly vertical lines and drooping curves) are explained in text. 
have been discovered. The standard model prediction for $S$ and $T$ curves down quite sharply in $T$ as $M_{H}$ is increased, quickly departing from the region allowed by the fit to electroweak data. (Useful analytic expressions for the contribution of a Higgs boson to $S$ and $T$ are given by [4.) However, if a small amount of triplet symmetry breaking is permitted, the agreement with the electroweak fit can be restored. As an example, a value of $V_{1,0} / v$ slightly smaller than $3 \%$ permits satisfactory agreement even for $M_{H}=1 \mathrm{TeV}$, as shown by the vertical line in the Figure.

If electroweak-symmetry-breaking vacuum expectation values are not due to a fundamental Higgs boson but rather to higher-dimension operators, one might well expect both Higgs doublet and Higgs triplet contributions, with their ratio indicating a geometric hierarchy of symmetry-breaking mass scales. (See 46, 47] for some early examples of this behavior.) One might then expect Higgs singlets of various types to have characteristic vacuum expectation values of $V_{0} \simeq v^{2} / V_{1,0} \simeq 246 \mathrm{GeV} / 0.03 \simeq 8$ $\mathrm{TeV}$. It is questionable whether the CERN Large Hadron Collider (LHC), with a total $p p$ center-of-mass energy of $14 \mathrm{TeV}$, could shed light on this mass scale.

What atomic-parity violation measurement would have a noticeable effect on the fit shown in Figure 1? The present error of \pm 0.8 on $Q_{W}(\mathrm{Cs})$ is equivalent to $\Delta S= \pm 1$. To match the error of \pm 0.15 on $S$ from the fits, one would have to determine $Q_{W}(\mathrm{Cs})$ a factor of between 6 and 7 more precisely than at present. The most significant $(>3 \sigma)$ discrepancies in present electroweak fits are (a) the difference between values of $\sin ^{2} \theta^{\text {eff }}$ measured using asymmetries of quarks and those using leptons 455, and (b) the the difference between directly measured $M_{W}$ values and those inferred from the neutral-current data of $\mathrm{NuTeV}$ [41]. Reduction of theoretical uncertainties associated with atomic physics calculations will be needed before one can claim similar discrepancies in atomic parity violation.

The need for determining $S$ independently of $T$ is highlighted by the Higgs-triplet example we have quoted. If a small Higgs-triplet contribution is present, one should be prepared to determine $S$ to an accuracy of better than \pm 0.1 if one wishes to pinpoint the Higgs boson mass via this indirect method. Of course, there is no substitute for direct searches, which the Fermilab Tevatron and the CERN Large Hadron Collider will provide in due course. It is also seen from Figure 1 that a minimal "technicolor" contribution of $\Delta S=0.2$ cannot be excluded at the $90 \%$ confidence-level limit if one is prepared to admit a Higgs-triplet contribution and a very heavy Higgs boson.

We thank Z. Luo for discussions regarding the $S$ and $T$ dependence of the NuTeV measurement, K. S. McFarland, O. P. Sushkov, and G. P. Zeller for helpful comments, and Michael Peskin for communicating the curves of Ref. [3] quoted in Fig. 1. This work was supported in part by the United States Department of Energy under Grant No. DE FG02 90ER40560. 


\section{References}

[1] S. L. Glashow, Nucl. Phys. 22, 579 (1961); S. Weinberg, Phys. Rev. Lett. 19, 1264 (1967); A. Salam, in Proceedings of the Eighth Nobel Symposium, edited by N. Svartholm (Almqvist and Wiksell, Stockholm; Wiley, New York, 1978), p. 367.

[2] W. J. Marciano, Brookhaven National Laboratory Report No. BNL-HET-00/04, hep-ph/0003181, to be published in Proceedings of MuMu99 - 5th International Conference on Physics Potential and Development of $\mu^{ \pm} \mu^{-}$Colliders, San Francisco, CA, Dec. 1999.

[3] M. E. Peskin and J. D. Wells, Stanford Linear Accelerator Center Report No. SLAC-PUB-8763, hep-ph/0101342, submitted to Phys. Rev. D.

[4] J. R. Forshaw, D. A. Ross, and B. E. White, University of Manchester report MC-TH-01/07, hep-ph/0107232 (unpublished).

[5] H.-J. He, C. T. Hill, and T. M. P. Tait, Univ. of Texas Report No. UTEXASHEP-01-013, hep-ph/0108041 (unpublished); H. J. He, N. Polonsky, and S. Su, Phys. Rev. D 64, 053004 (2001).

[6] B. Dobrescu and C. T. Hill, Phys. Rev. Lett. 81, 2634 (1998).

[7] H. Collins, A. K. Grant, and H. Georgi, Phys. Rev. D 61, 055002 (2000).

[8] M. E. Peskin, "Interpretation of Precision Electroweak Data, or Should We Really Believe There is a Light Higgs Boson?", seminar at Snowmass 2001 Workshop, July, 2001 (unpublished).

[9] M. Peskin and T. Takeuchi, Phys. Rev. Lett. 65, 964 (1990); Phys. Rev. D 46, 381 (1992).

[10] C. S. Wood et al., Science 275, 1759 (1997).

[11] S. C. Bennett and C. E. Wieman, Phys. Rev. Lett. 82, 2484 (1999).

[12] M. J. D. Macpherson, K. P. Zetie, R. B. Warrington, D. N. Stacey, and J. P. Hoare, Phys. Rev. Lett. 67, 2784 (1991).

[13] D. M. Meekhof, P. Vetter, P. K. Majumder, S. K. Lamoureaux, and E. N. Fortson, Phys. Rev. Lett. 71, 3442 (1993).

[14] P. A. Vetter, D. M. Meekhof, P. K. Majumder, S. K. Lamoreaux, and E. N. Fortson, Phys. Rev. Lett. 74, 2658 (1995).

[15] N. H. Edwards, S. J. Phipp, P. E. G. Baird, and S. Nakayama, Phys. Rev. Lett. 74, 2654 (1995). 
[16] W. Marciano and J. L. Rosner, Phys. Rev. Lett. 65, 2963 (1990); 68, 898(E) (1992).

[17] P. G. H. Sandars, J. Phys. B 23, L655 (1990).

[18] J. L. Rosner, Phys. Rev. D 42, 3107 (1990).

[19] J. L. Rosner, Phys. Rev. D 53, 2724 (1996).

[20] J. L. Rosner, Comments on Nucl. Part. Phys. 22, 205 (1998).

[21] J. L. Rosner, Phys. Rev. D 61, 016006 (1999).

[22] V. A. Dzuba, V. V. Flambaum, and O. P. Sushkov, Phys. Lett. A 141, 147 (1989); S. A. Blundell, W. R. Johnson, and J. Sapirstein, Phys. Rev. Lett. 65, 1411 (1990); Phys. Rev. D 45, 1602 (1992).

[23] V. A. Dzuba, V. V. Flambaum, P. G. Silvestrov, and O. P. Sushkov, J. Phys. B 20, 3297 (1987).

[24] T. Takeuchi, W. Loinaz, and A. Grant, Virginia Tech report VPI-IPPAP-9903, hep-ph/9904207, presented by T. Takeuchi at Hadron Collider Physics 13, Mumbai, India, January 14-20, 1999 (unpublished).

[25] R. Casalbuoni, S. De Curtis, D. Dominici, and R. Gatto, Phys. Lett. B 460, 135 (1999).

[26] J. Erler and P. Langacker, Phys. Lett. B 456, 68 (1999).

[27] P. G. Langacker, R. Robinett, and J. L. Rosner, Phys. Rev. D 30, 1470 (1984).

[28] D. London and J. L. Rosner, Phys. Rev. D 34, 1530 (1986).

[29] CDF Collaboration, F. Abe et al., Phys. Rev. Lett. 79, 2192 (1997).

[30] A. Derevianko, Phys. Rev. Lett. 85, 1618 (2000); University of Nevada at Reno Report, physics/0108033 (unpublished).

[31] M. G. Kozlov, S. G. Porsev, and I. I. Tupitsyn, Phys. Rev. Lett. 86, 3260 (2001).

[32] V. A. Dzuba, C. Harabati, W. R. Johnson, and M. S. Safronova, Phys. Rev. A 63, 044103 (2001).

[33] A. I. Milstein and O. P. Sushkov, preprint hep-ph/0109257 (unpublished).

[34] G. Breit, Phys. Rev. 34, 553 (1929); 36, 383 (1930); 39, 616 (1932).

[35] M. Davier M and A. Höcker, Phys. Lett. B 419, 419 (1998); 435, 427 (1998).

[36] M. Veltman, Nucl. Phys. B123, 89 (1977); Acta Phys. Polonica B8, 475 (1977). 
[37] P. Sikivie, L. Susskind, M. B. Voloshin, and V. Zakharov, Nucl. Phys. B173, 189 (1980).

[38] D. C. Kennedy and P. G. Langacker, Phys. Rev. Lett. 65, 2967 (1990); 66, 395(E) (1991).

[39] J. L. Rosner, Enrico Fermi Institute Report No. EFI01-34, hep-ph/0108195, lectures at the 55th Scottish Universities' Summer School in Particle Physics, St. Andrews, Scotland, August 7-23, 2001. Proceedings to be published by the Institute of Physics (U.K.).

[40] M. Swartz, lecture at Snowmass 2001 Workshop, transparencies available at http://pha.jhu.edu/ morris/higgs.pdt.

[41] NuTeV Collaboration, G. P. Zeller et al., preprint hep-ex/0110059, submitted to Phys. Rev. Letters.

[42] NuTeV Collaboration, G. P. Zeller et al., preprint hep-ex/9906024, published in Proceedings of American Physical Society (APS) Meeting of the Division of Particles and Fields (DPF 99), Los Angeles, CA, 5-9 Jan 1999.

[43] P. G. H. Sandars and B. W. Lynn, J. Phys. B 27, 1469 (1994).

[44] D. Charlton, plenary talk at International Europhysics Conference on High Energy Physics, Budapest, Hungary, July 12-18, 2001.

[45] LEP Electroweak Working Group; see web page http://lepewwg.web.cern.ch/LEPEWWG for periodic updates.

[46] C. N. Leung, R. W. Robinett, and J. L. Rosner, in Proceedings of the Neutrino Mass Mini-Conference, Telemark, WI., Sept. 23-25, 1982, edited by Vernon Barger and David Cline, American Institute of Physics, New York, 1983, p. 202.

[47] C. N. Leung and J. L. Rosner, Phys. Rev. D 29, 2132 (1984). 